\title{
Research of Herb-Partitioned Moxibustion for Primary Dysmenorrhea Patients Based on the LC-MS Metabonomics
}

\author{
Yu-xia Ma, ${ }^{1}$ Xing-yue Yang, ${ }^{1}$ Gang Guo, ${ }^{2}$ Dong-qing Du, ${ }^{3}$ Yan-pu Yu, ${ }^{1}$ and Shu-zhong Gao \\ ${ }^{1}$ Shandong University of Traditional Chinese Medicine, Shandong 250355, China \\ ${ }^{2}$ Qilu Hospital of Shandong University, Shandong 250012, China \\ ${ }^{3}$ Jinan Hospital of Traditional Chinese Medicine, Shandong 250012, China
}

Correspondence should be addressed to Shu-zhong Gao; gaoshuzhong@163.com

Received 16 November 2014; Revised 11 April 2015; Accepted 16 April 2015

Academic Editor: Jian Kong

Copyright (C) $2015 \mathrm{Yu}$-xia Ma et al. This is an open access article distributed under the Creative Commons Attribution License, which permits unrestricted use, distribution, and reproduction in any medium, provided the original work is properly cited.

\begin{abstract}
Objective. To explore the efficacy and mechanism of primary dysmenorrhea patients were treated with herb-partitioned moxibustion through metabonomics. Methods. 20 patients with primary dysmenorrhea were randomized into two groups, separately treated with herb-partitioned moxibustion at CV8 (shenque) and acupuncture at SP6 (sanyinjiao). After three menstrual cycles' treatment, the intensity of menstrual pain using VAS and the changes of metabolites of plasma using LCMS were observed. Results. The VAS of two groups decreased with different descending range. Herb-partitioned moxibustion upregulated $20 \alpha$-dihydroprogesterone, pregnenolone, prostaglandin $\mathrm{E}_{2}$ and $\gamma$-aminobutyric acid and downregulated the content of estrone and prostaglandin $\mathrm{H}_{2}$, while acupuncture upregulated pregnenolone and $20 \alpha$-dihydroprogesterone and downregulated 2-methoxyestradiol-3-methylether, 15-hydroxyeicosatrienoic acid and 6-keto-prostaglandin. Discussion. It was effective in relieving the abdominal pain by these two therapies. Herb-partitioned moxibustion is superior to acupuncture for primary dysmenorrhea, which could be related to regulating the endocrine hormone.
\end{abstract}

\section{Introduction}

Primary dysmenorrhea (PD) is the painful menstrual cramps of uterine in woman during menstruation without detectable organic disease [1]. Most of the PD patients develop the typical symptoms such as crampy pelvic pain with pain radiating to the lower back or anterior thigh, nausea, vomiting, diarrhea, headache, fatigue, nervousness, and dizziness before or at the onset of menses and lasting one to three days [2]. It is the most common gynecologic complaint especially among adolescent women with estimates ranging from 40 to 50 percent [3], which has disturbed their routine activities and may lead to infertility in severe cases [4, 5]. The main treatment of modern medicine is oral spasmolysis analgesic, acyeterion, and calcium ion antagonist. Nowadays, nonsteroid anti-inflammatory drug (NSAIDs), like Ibuprofen, indomethacin, and chlorine acid, is the firstline drugs for the treatment of primary dysmenorrhea [6], which is to reduce the accumulation of prostaglandin and then alleviate spasmodic contraction of the uterus caused by prostaglandins, by inhibiting the COX-2 enzymes activity [7]. But at the same time COX-1 would also be inhibited and then causes adverse reactions such as nausea, diarrhea, and headache [8]. Recent studies have shown that cardiovascular and cerebrovascular diseases would also be induced through chronic use of these drugs, such as unstable angina, acute myocardial infarction, and venous thromboembolism [9].

Therefore, it is necessary to seek a new effective therapy to treat PD. Traditional Chinese medicine (TCM), such as acupuncture [10] and herbal medicine [11], has been evaluated through a number clinical trials, suggesting preliminary evidence of efficacy for PD. Herb-partitioned moxibustion (HPM) is a characteristic external therapy in TCM, which is widely used to treat diseases in China. The preliminary work of our research team has proved that HPM can immediately relieve menstrual pain [12]. But its mechanism is still unclear. Therefore, it needs further study.

Metabonomics indicates the overall physiological status by analyzing the dynamic changes of low molecular weight metabolites [13]. The pathogenesis of diseases and 


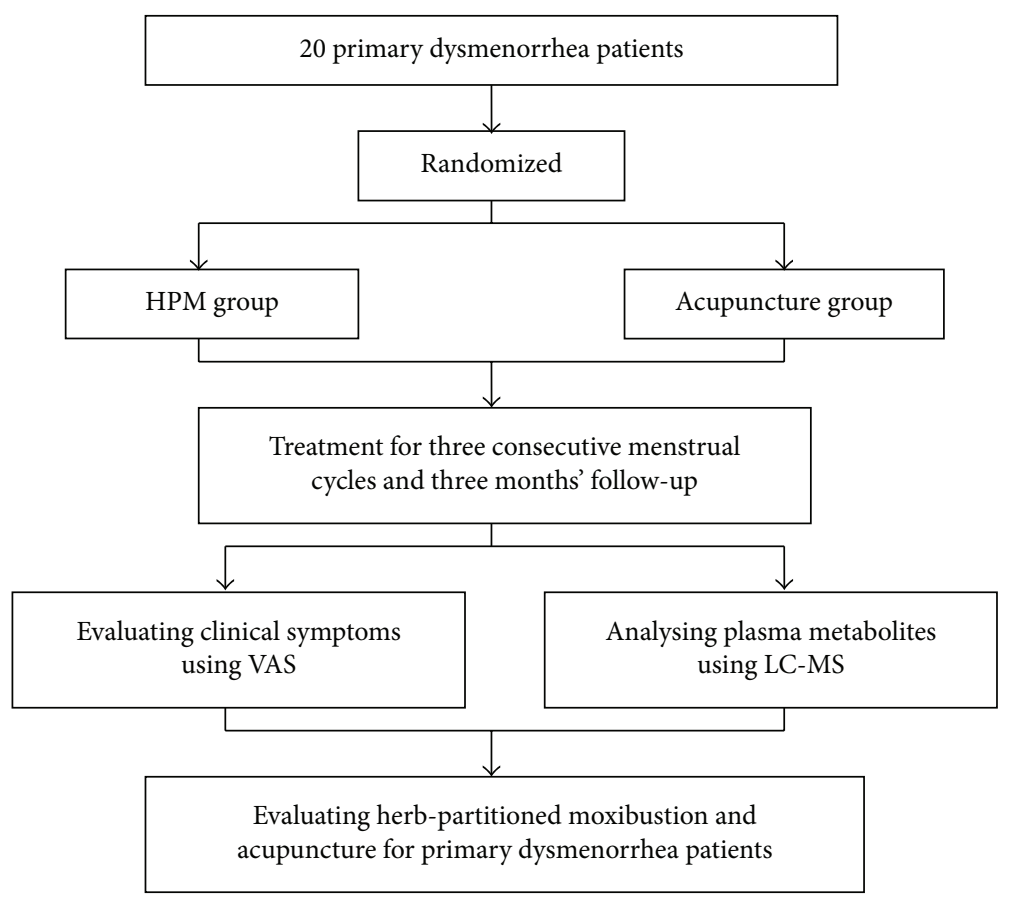

FIGURE 1: Flow chart of the study.

the action mechanisms of therapy would be elucidated by identifying the biomarkers and analyzing the metabolic pathway. Recently, metabonomics has attracted an interest for biomarker discovery and for assessing holistic therapeutic effects of many TCMs $[14,15]$. Liquid chromatographymass spectrometry (LC-MS) has demonstrated being the frequently used analytical technologies in metabonomicsbased studies [16,17], allowing expanding the number of metabolites that can be comprehensively covered in experiments.

In this study, our aims are (1) to verify and to compare the clinical efficacy of HPM and acupuncture for PD patients; (2) to preliminarily elucidate the possible mechanisms of HPM for PD by using a metabonomics analysis.

\section{Methods}

2.1. Patient Selection. Patients were recruited from the outpatients of Affiliated Hospital of Shandong University of Traditional Chinese Medicine between January 2011 and December 2012. The study protocol was approved by the Ethics Committee of Shandong University of Traditional Chinese Medicine Affiliated Hospital in 2010 (Registration number 20100137). All the volunteers provided informed consent.

Eligible patients met the following inclusion criteria: (1) the diagnostic criteria of primary dysmenorrhea in the Primary Dysmenorrhea Consensus Guideline [18]; (2) age from 16 to 35 years without history of delivery; (3) being with normal menstrual cycle (28 \pm 7 days); (4) VAS (visual analogue scale) scoring more than $40 \mathrm{~mm}$; (5) no oral administration of any analgesic and other hormones such as oral contraceptive pill or acceptance of other therapies on menstrual cycle before the trial and during the experimental period. The following main criteria for exclusion were applied: women with secondary dysmenorrhea caused by endometriosis, uterine myoma, endometrial polyps, pelvic inflammatory disease, and other gynecological problems; pregnant and lactating women; women with other serious illnesses and unsuited for acupuncture.

2.2. Randomization and Blinding. Randomization was performed by an independent statistician through generating allocation numbers based on a random number creation system. 20 eligible patients were randomly assigned into two groups (HPM group and acupuncture group), each group with 10 patients, respectively (Figure 1). Double-blind trial could not be performed in this study. However, data collectors and data statisticians were all blinded to treatment assignments.

2.3. Interventions. The points used in this study were located according to Chinese National Criteria for Points Location (GB12346-90). Acupuncture interventions were performed by the same acupuncturist who had the Chinese medicine practitioner license from the Ministry of Health of the People's Republic of China throughout the entire study. Sterile disposable acupuncture needles (Huatuo $0.30 \times 40 \mathrm{~mm}$, made in Suzhou Medical Instruments Factory) were used. The medicinal herbs used were purchased from Jianlian Medicine Company (Jinan, China) and were authenticated by professor of pharmacognosy. The herbs mainly included wuzhuyu, bai shao, ruxiang, moyao, yanhusuo, bing pian, and wulingzhi, which were smashed by ultrafine grinder into pulverata. 


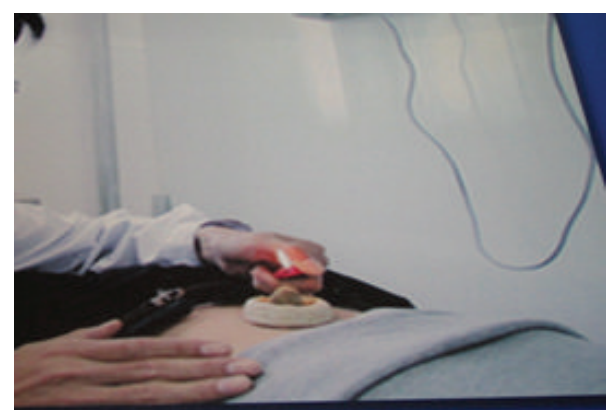

Figure 2: HPM for PD patients in clinic. A bowl made by dough with a hole (diameter $2 \mathrm{~cm}$, depth $2 \mathrm{~cm}$ ) in the middle was placed on CV8. Medicamental pulverata (about $8-10 \mathrm{~g}$ ) was filled in the hole. Then a burning moxa cone (diameter $2 \mathrm{~cm}$, height $2 \mathrm{~cm}$ ) was put on the medicamental pulverata and changed till it burned out. Ten moxa cones were used, about 2 hours, during each treatment time.

2.3.1. HPM Group. The herbs were mixed in proportions and were shattered into medicamental pulverata by pulverizer. Participants were asked to lay on their back, and then the CV8 (shenque, in the middle of navel) and its surrounding skin were disinfected using $75 \%$ alcohol. A bowl made by dough with a hole (diameter $2 \mathrm{~cm}$, depth $2 \mathrm{~cm}$ ) in the middle was placed on CV8. Medicamental pulverata (about 8-10 g) was filled in the hole. Then a burning moxa cone (diameter $2 \mathrm{~cm}$, height $2 \mathrm{~cm}$ ) was put on the medicamental pulverata and changed till it burned out (Figure 2). Ten moxa cones were used, about 2 hours, during each treatment time. At the end of the treatment, the medicamental pulverata was sealed with adhesive tape and washed 24 hours later.

2.3.2. Acupuncture Group. The participants were punctured perpendicularly at bilateral SP6 (sanyinjiao, 3 cun above the medial malleolus, at the posterior border of the medial aspect of the tibia) to a depth of $2.5-4.0 \mathrm{~cm}$ in supine position. Manipulating needling, lifting-thrusting and twirling for one minute, were used to induce deqi. And then the needles were retained for 15 minutes.

The performer started the intervention 7 days before menses once a day by acupuncture and once every three days by moxibustion until menstruating. Participants received the treatment for three consecutive menstrual cycles and three months' follow-up. After the course, we analyzed the outcomes. During the treatment period, the participants could take aspirin effervescent tablets orally distributed by performers when they could not endure menstrual pain (VAS $\geq 80$ ), which would be recorded by the participants and dosage by the performers.

\subsection{Outcome Measures}

2.4.1. The Primary Outcome Measures. The VAS was used to measure the abdominal pain of participants from baseline to follow-up recorded by another performer.

2.4.2. The Secondary Outcome Measures. We utilized LCMS to analyse plasma metabolites. 20 participants waited for one menstrual cycle without treatment before cycle 1 . Blood specimens were collected in the first or second day during the menstruation of the waiting month and the first month after treatment course. The blood specimen was put into anticoagulant tube for centrifugation for $5 \mathrm{~min}$ with $5000 \mathrm{r} \cdot \mathrm{min}^{-1}$ and then the plasma was kept at $-28^{\circ} \mathrm{C}$ in reserve. The plasma specimen would be taken out before the testing and room temperature melting and then was processed with centrifugation for 5 minutes with $10800 \mathrm{rpm}$ after being precipitated by acetonitrile first. Take the supernatant liquid for testing. LC-MS analysis was performed on Agilent 1290 Infinity LC system coupled to Agilent 6530 Accurate-Mass Quadrupole Time-of-Flight (Q-TOF) mass spectrometer (Agilent, USA). Chromatographic separations were performed on an ACQUITY UPLCTM BEH C18 column $(2.7 \mu \mathrm{m}, 3.0 * 50 \mathrm{~mm})$, flow rate: $0.4 \mathrm{~mL} / \mathrm{min}$, temperature in the injection chamber: $4^{\circ} \mathrm{C}$, column temperature: $35^{\circ} \mathrm{C}$, and sample size: $5 \mu \mathrm{L}$. An electrospray ionization source (ESI) interface was used and was set in both positive and negative modes so as to monitor as many ions as possible. The following parameters of MS were employed: electrospray ion source, mass scan range: 50-1100 Da, capillary voltage: $3500 \mathrm{~V}$, quadrupole rod temperature: $100^{\circ} \mathrm{C}$, fragmentor: $175 \mathrm{~V}$, nebulizer pressure: $35 \mathrm{psig}$, gas temperature: $350^{\circ} \mathrm{C}$, and gas flow: $10 \mathrm{~L} / \mathrm{min}$.

2.5. Statistical Analysis. The raw LC-MS data from metabolic profiling were pretreated following the procedure described. We analyzed the data using Masshunter quantitative data analysis software to extract molecular peak feature of data after removing noise, in addition to the internal standard, peak, and peak alignment, normalized, and then using Simca$\mathrm{P}$ (version 11.5) software to proceed principal component analysis (PCA) and partial least square-discriminant analysis (PLS-DA), and applying VIP (very important in the projection) data to find out the characteristic metabolites. The related metabolic pathways information was from the HMDB and KEGG software. We used SPSS (version 17.0) to analyse the data; measurement data was presented by mean and standard error (SE), using $t$-test. $P$ values reported in this paper are two-sided and $P$ values of $<0.05$ were considered statistically significant.

\section{Results}

3.1. Menstrual Abdominal Pain with VAS Scores. From Figure 3, we could see that the VAS value of both two groups reduced gradually, and there was statistical significance compared to cycle 2, cycle 3, and follow-up with the baseline $(P<0.01)$. The comparison of VAS score between the two groups showed that there was statistical significance at cycle 3 and follow-up $(P<0.01)$. No difference was identified between Group A and Group B at the baseline, cycle 1, and cycle $2(P>0.05)$.

3.2. Analysing the Potential Biomarkers. The metabolomics figures of two groups were shown from Figures 4-7. Figures 4 and 5 show typical LC-MS total ion current (TIC) 
TABLE 1: The main probable materials of two groups.

\begin{tabular}{|c|c|c|c|c|c|c|c|}
\hline Group & ESI & $\begin{array}{c}\text { Monoisotopic } \\
\text { molecular weight }\end{array}$ & Formula & Metabolites & Related pathway & VIP & Changed fold ${ }^{\mathrm{a}}$ \\
\hline A & + & 270.1620 & $\mathrm{C}_{18} \mathrm{H}_{22} \mathrm{O}_{2}$ & Estrone & Steroid hormone biosynthesis & 1.56605 & $\downarrow$ \\
\hline A & + & 316.2402 & $\mathrm{C}_{21} \mathrm{H}_{32} \mathrm{O}_{2}$ & $20 \alpha$-Dihydroprogesterone & Steroid hormone biosynthesis & 1.30279 & $\uparrow$ \\
\hline A & + & 316.2402 & $\mathrm{C}_{21} \mathrm{H}_{32} \mathrm{O}_{2}$ & Pregnenolone & Steroid hormone biosynthesis & 1.30279 & $\uparrow$ \\
\hline A & + & 352.2250 & $\mathrm{C}_{20} \mathrm{H}_{32} \mathrm{O}_{5}$ & Prostaglandin $\mathrm{E}_{2}$ & Steroid hormone biosynthesis & 1.47588 & $\uparrow$ \\
\hline A & + & 352.2250 & $\mathrm{C}_{20} \mathrm{H}_{32} \mathrm{O}_{5}$ & Prostaglandin $\mathrm{H} 2$ & Arachidonic acid metabolism & 1.47588 & $\downarrow$ \\
\hline A & + & 103.0633 & $\mathrm{C}_{4} \mathrm{H}_{9} \mathrm{NO}_{2}$ & Gamma-Aminobutyric acid & GABAergic synapse & 1.3201 & $\uparrow$ \\
\hline A & - & 284.1412 & $\mathrm{C}_{18} \mathrm{H}_{20} \mathrm{O}_{3}$ & 16-Oxoestrone & Steroid hormone biosynthesis & 1.10038 & $\downarrow$ \\
\hline B & + & 316.2038 & $\mathrm{C}_{20} \mathrm{H}_{28} \mathrm{O}_{3}$ & 2-Methoxyestradiol-3-methylether & Steroid hormone biosynthesis & 1.59796 & $\downarrow$ \\
\hline B & + & 316.2402 & $\mathrm{C}_{21} \mathrm{H}_{32} \mathrm{O}_{2}$ & $20 \alpha$-Dihydroprogesterone & Steroid hormone biosynthesis & 1.12801 & $\uparrow$ \\
\hline B & + & 316.2402 & $\mathrm{C}_{21} \mathrm{H}_{32} \mathrm{O}_{2}$ & Pregnenolone & Steroid hormone biosynthesis & 1.12801 & $\uparrow$ \\
\hline B & + & 322.25 & $\mathrm{C}_{20} \mathrm{H}_{34} \mathrm{O}_{3}$ & 15-Hydroxyeicosatrienoic acid & Arachidonic acid metabolism & 1.47681 & $\downarrow$ \\
\hline B & - & 370.2356 & $\mathrm{C}_{20} \mathrm{H}_{34} \mathrm{O}_{6}$ & 6-Keto-prostaglandin & Arachidonic acid metabolism & 2.46181 & $\downarrow$ \\
\hline
\end{tabular}

${ }^{a}$ compared to the content before treatment. Arrow $(\uparrow)$ indicated relative increasing in signal. Arrow $(\downarrow)$ indicated relative decreasing in signal. Differences with $P<0.05$ were considered significant.

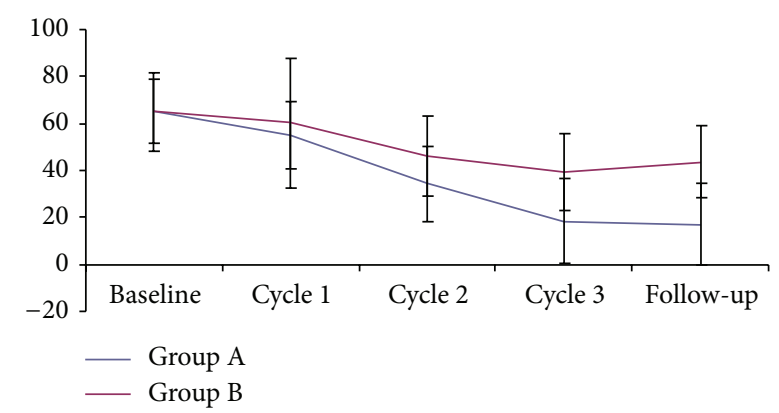

FIGURE 3: The VAS scores of each cycle.

chromatograms of a plasma sample in positive ionization mode (Figure 4) and negative ionization mode (Figure 5). Compared to Figures 4(a) and 4(b), Figures 5(a) and 5(b), the TIC of group HPM and group acupuncture had obvious differences. In the PLS-DA plots (Figures 6 and 7), these two groups could also be well distinguished. Therefore, we deduced that the metabolite changes after treatment were different.

Figure 8 expressed the variation trend of metabolite, reducing from left to right. With VIP $>1$ as a standard (where a VIP value of $>1$ is regarded as significant), we found 6950 kinds of ion information of group HPM; 7 metabolites were considered as the potential biomarkers. In group acupuncture, we found 4460 kinds of ion information, and 5 metabolites were considered as the potential biomarkers. It was shown in Table 1. In this study, the HPM upregulated the 20 $\alpha$-dihydroprogesterone, pregnenolone, prostaglandin $\mathrm{E}_{2}$, and gamma-aminobutyric acid and downregulated estrone, 16-oxoestrone, and prostaglandin $\mathrm{H}_{2}$. Acupuncture upregulated pregnenolone, and $20 \alpha$-dihydroprogesterone and downregulated 2-methoxyestradiol-3-methylether, 15hydroxyeicosatrienoic acid, and 6-keto-prostaglandin. However, the remaining biomarkers (data not shown) were unidentifiable due to insufficient intensity for MS experiments or the restrictions of current metabolite databases.

\section{Discussion}

In the study, these results suggested that HPM could improve the menstrual pain of PD. LC-MS was used to investigate the plasma metabolic profile associated with PD. Seven potential biomarkers (20 $\alpha$-dihydroprogesterone, pregnenolone, prostaglandin $\mathrm{E}_{2}$, estrone, 16-oxoestrone, and prostaglandin $\mathrm{H}_{2}$ ) connected with PG, estrogen, and progestin have been found. The cramps of uterine in $\mathrm{PD}$ are thought to be caused by excessive production of prostaglandins (PG) and leukotrienes, which mediate hyperalgesia and cause vasoconstriction, ischemia, and myometrial contraction [19]. Moreover, the excess of PG would lead to cardiovascular and gastrointestinal symptoms [20]. It is indicated that the abnormal level of estrogen/progestin and immunologic function would lead to an excess of PG $[21,22]$. Further investigating the intervening mechanisms of HPM for PD, we found that most of the potential biomarkers involved in metabolic processes were related to steroid hormone biosynthesis. In other words, HPM can regulate the internal secretion of PD patients, which is mostly compatible with the results of our previous study [12]. In previous study, we have found that patients with HPM showed significant differences after treatment in the level of estradiol, progesterone, and prostaglandin using chemoimmunology therapy. In addition, another potential biomarker, gamma-aminobutyric acid, takes part in analgesia in central nervous system that can increase the pain threshold. Therefore, gamma-aminobutyric acid increasing could alleviate pain. Furthermore, HPM can regulate arachidonic acid metabolism, producing PG and leukotrienes through the function of cyclooxygenase and lipoxygenase.

According to statistics, SP6 (sanyinjiao) is the most common selected acupoint in PD trials [23]. Therefore, we choose acupuncture at SP6 as the control group to 


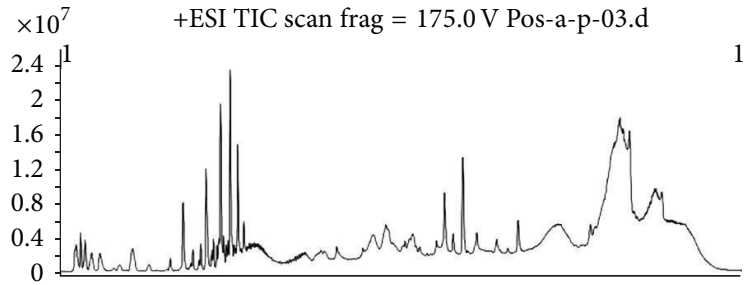

(a)

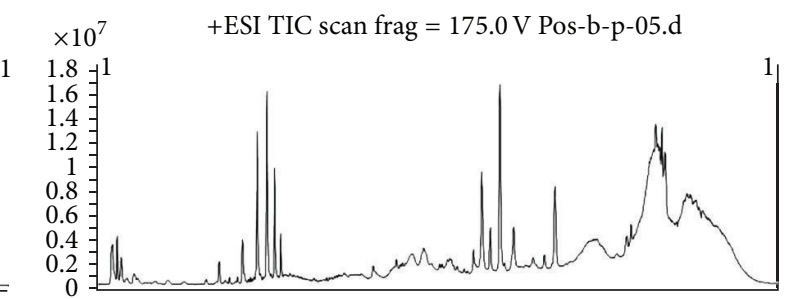

(b)

FIgURE 4: The TIA of two groups after treatment in ESI ${ }^{+}$mode: (a) HPM group; (b) acupuncture group. Group HPM and group acupuncture had obvious differences.

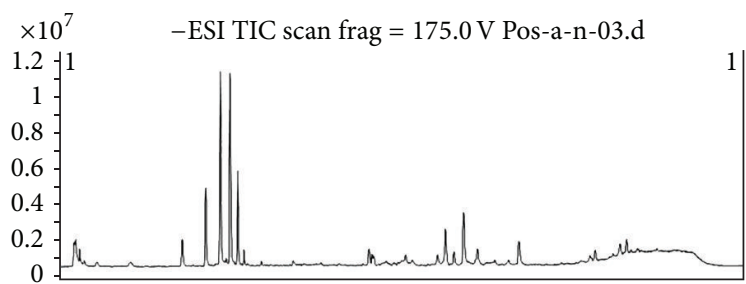

(a)

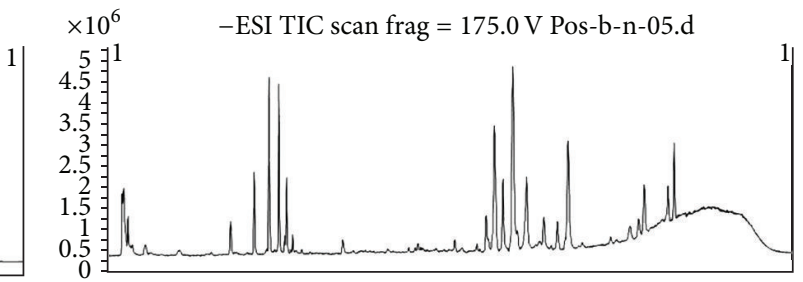

(b)

FIGURE 5: The TIA of two groups after treatment in $\mathrm{ESI}^{-}$mode: (a) HPM group; (b) acupuncture group. Group HPM and group acupuncture had obvious differences.

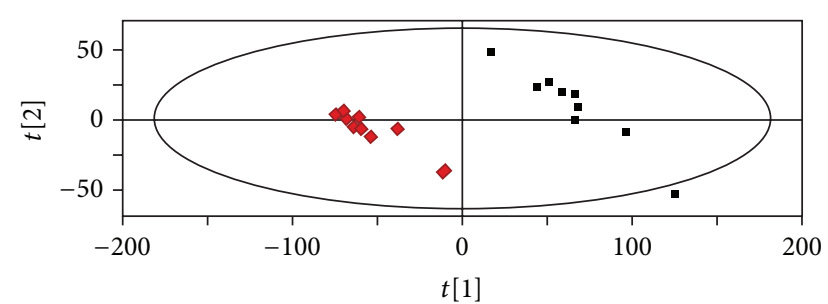

- Class 1

- Class 1

Figure 6: The PLS-DA plot from group HPM before and after treatment. Class 1: before treatment; class 2: after treatment.

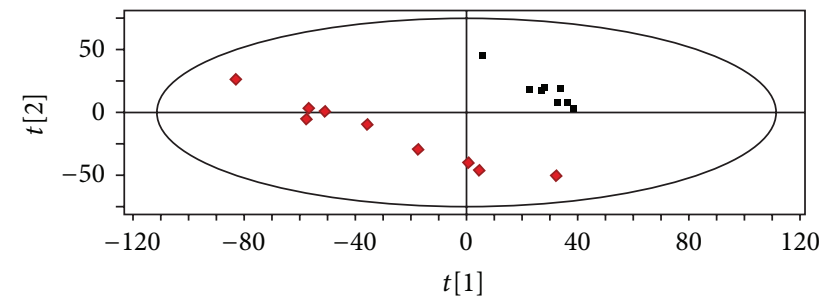

FIGURE 7: The PLS-DA plot from group acupuncture before and after treatment. Class 1: before treatment; class 2: after treatment.

demonstrate the clinical efficacy of HPM. From Figure 3, HPM has better curative effect in reducing the menstrual pain of PD patients, with stable efficacy in follow-up of three months. Meanwhile, the metabolite profiles of SP6 group showed 5 potential biomarkers (2-methoxyestradiol3-methylether, $20 \alpha$-dihydroprogesterone, pregnenolone, 15hydroxyeicosatrienoic acid, and 6-keto-prostaglandin), three of which are the same as HPM group. One of the other two potential biomarkers, 2-methoxyestradiol-3-methylether, is also related with steroid hormone biosynthesis. And 15-hydroxyeicosatrienoic acid revealed metabolic pathway (arachidonic acid metabolism) is mentioned above in group HPM. Therefore, the regulating internal secretion might also be involved in the intervening mechanism of acupuncture at SP6 for PD.

Compared with HPM and acupuncture at SP6 for PD, the VAS score shows that both two therapies can relieve menstrual pain, but the former one has better clinical efficiency. Using LC-MS, we find the two therapies express the changes mainly of endocrine hormone to regulate the internal secretion. But HPM has wider regulation than acupuncture comparing the metabolites. The group of HPM is better than the group of acupuncture at SP6 in regulating the estrone and prostaglandin level. Through our previous work, CV8 (shenque) is associated with five $z a n g$ and six $f u$ according to TCM theory. It is regarded as congenital foundation and the pivot of yin-yang balance. It is also close to pelvic cavity in anatomical position. Furthermore, it is easy for the effective constituent of herbs penetrating into body due to the thin skin around it. The main efficacy of the herbs used in our study is warming and activating meridian, promoting $q i$ circulation to relieve pain. The heat stimulation of moxibustion could accelerate the penetration of the herbs into human body. Therefore, HPM, combining the heat stimulation, herbal stimulation, and acupoint stimulation together, is suitable for gynecological diseases especially functional diseases such as PD.

However, whether HPM is superior to acupuncture or not is not concluded in this study because of a small sample experiment. It merits further study. Besides, on account of 


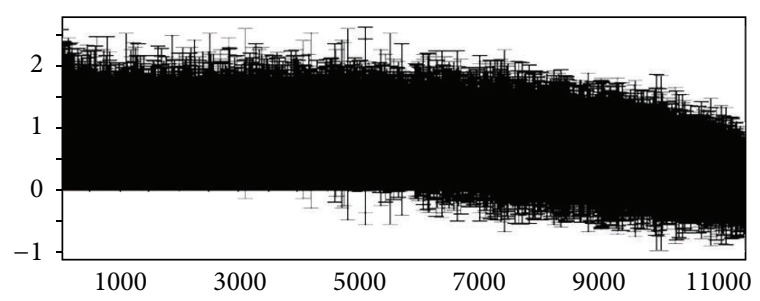

(a)

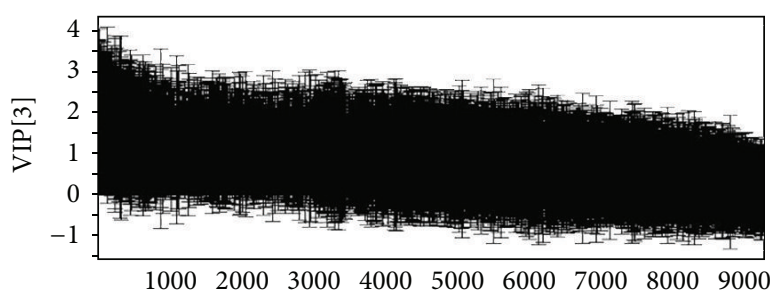

(b)

FIGURE 8: The VIP scores of PLS of two groups: (a) HPM group; (b) acupuncture group. The variation trend of metabolite, reducing from left to right $(P<0.05)$.

the metabonomics method, the metabolism of the whole body cannot be reflected fully. The further study on the mechanisms of the changes of metabolite of PD patients should expand the sample size and then, combined with molecular biology, imageology and other research methods to explore the mechanism of acupuncture and moxibustion for PD.

\section{Conclusion}

The present study plays an important role in providing an effective alternative for the treatment of $\mathrm{PD}$ and further investigating the mechanisms of HPM for PD. HPM can relieve menstrual pain of $\mathrm{PD}$ patients possibly through regulating internal secretion. On account of the present limitation, further study is being carried out in our laboratory.

\section{Conflict of Interests}

The authors declare that they have no competing interests.

\section{Authors' Contribution}

Yu-xia Ma and Xing-yue Yang and their affiliations contribute equally to this paper.

\section{Acknowledgment}

This study was supported by a grant from the National Natural Science Foundation Item (Grant no. 30973789).

\section{References}

[1] M. Y. Dawood, "Primary dysmenorrhea: advances in pathogenesis and management," Obstetrics and Gynecology, vol. 108, no. 2, pp. 428-441, 2006.

[2] T. I. J. Hillen, S. L. Grbavac, P. J. Johnston, J. A. Y. Straton, and J. M. F. Keogh, "Primary dysmenorrhea in young Western Australian women: Prevalence, impact, and knowledge of treatment," Journal of Adolescent Health, vol. 25, no. 1, pp. 40-45, 1999.

[3] C. Banikarim, M. R. Chacko, and S. H. Kelder, "Prevalence and impact of dysmenorrhea on hispanic female adolescents," Archives of Pediatrics and Adolescent Medicine, vol. 154, no. 12, pp. 1226-1229, 2000.
[4] D. A. Holtzman, K. L. Petrocco-Napuli, and J. R. Burke, "Prospective case series on the effects of lumbosacral manipulation on dysmenorrhea," Journal of Manipulative and Physiological Therapeutics, vol. 31, no. 3, pp. 237-246, 2008.

[5] S. E. Daniels, S. Torri, and P. J. Desjardins, "Valdecoxib for treatment of primary dysmenorrhea: a randomized, doubleblind comparison with placebo and naproxen," Journal of General Internal Medicine, vol. 20, no. 1, pp. 62-67, 2005.

[6] C. Bilir, N. Akdemir, D. Çolak, and H. Cinemre, "Electrocardiographic findings in patients with primary dysmenorrhea," The American Journal of the Medical Sciences, vol. 343, no. 1, pp. 2729, 2012.

[7] I. Şahin, F. Saraçoğlu, Y. Kurban, and B. Türkkani, "Dysmenorrhea treatment with a single daily dose of rofecoxib," International Journal of Gynecology and Obstetrics, vol. 83, no. 3, pp. 285-291, 2003.

[8] Federação Brasileira das Associações de Ginecologia E Obstetrícia, "Primary dysmenorrhea: treatment," Revista da Associação Médica Brasileira, vol. 59, no. 5, pp. 413-419, 2013.

[9] American College of Rheumatology Subcommittee on Osteoarthritis Guidelines, "Recommendations for the medical management of osteoarthritis of the hip and knee: 2000 update," Arthritis \& Rheumatism, vol. 43, no. 9, pp. 1905-1915, 2000.

[10] Y.-X. Ma, X.-N. Ye, C.-Z. Liu et al., "A clinical trial of acupuncture about time-varying treatment and points selection in primary dysmenorrhea," Journal of Ethnopharmacology, vol. 148, no. 2, pp. 498-504, 2013.

[11] H. Y. Chen, Y. H. Lin, I. H. Su, Y. C. Chen, S. H. Yang, and J. L. Chen, "Investigation on chinese herbal medicine for primary dysmenorrhea: implication from a nationwide prescription database in taiwan," Complementary Therapies in Medicine, vol. 22, no. 1, pp. 116-125, 2014.

[12] Z. Xue, C. Z. Liu, S. Z. Gao, and Y. X. Ma, "The herbalpartitioned moxibustion for primary dysmenorrhea and it's impact on reproductive endocrinal function of patients," Zhongguo Zhen Jiu, vol. 34, no. 3, pp. 209-212, 2014.

[13] J. K. Nicholson and I. D. Wilson, "Understanding 'global' systems biology: metabonomics and the continuum of metabolism," Nature Reviews Drug Discovery, vol. 2, no. 8, pp. 668-676, 2003.

[14] X. Wang, B. Yang, A. Zhang, H. Sun, and G. Yan, "Potential drug targets on insomnia and intervention effects of Jujuboside A through metabolic pathway analysis as revealed by UPLC/ESISYNAPT-HDMS coupled with pattern recognition approach," Journal of Proteomics, vol. 75, no. 4, pp. 1411-1427, 2012. 
[15] G. G. Tan, W. T. Liao, X. Dong et al., "Metabonomic profiles delineate the effect of traditional Chinese medicine Sini decoction on myocardial infarction in rats," PLoS ONE, vol. 7, no. 4, Article ID e34157, 2012.

[16] M. Sieber, S. Wagner, E. Rached, A. Amberg, A. Mally, and W. Dekant, "Metabonomic study of ochratoxin A toxicity in rats after repeated administration: phenotypic anchoring enhances the ability for biomarker discovery," Chemical Research in Toxicology, vol. 22, no. 7, pp. 1221-1231, 2009.

[17] R. Williams, E. M. Lenz, A. J. Wilson et al., "A multi-analytical platform approach to the metabonomic analysis of plasma from normal and zucker (fa/fa) obese rats," Molecular BioSystems, vol. 2, no. 3-4, pp. 174-183, 2006.

[18] G. Lefebvre, O. Pinsonneault, V. Antao et al., "Primary dysmenorrhea consensus guideline," Journal of Obstetrics and Gynaecology Canada, vol. 27, no. 12, pp. 1117-1146, 2005.

[19] Z. Harel, "A contemporary approach to dysmenorrhea in adolescents," Pediatric Drugs, vol. 4, no. 12, pp. 797-805, 2002.

[20] J. R. Dingfelder, "Primary dysmenorrhea treatment with prostaglandin inhibitors: a review," American Journal of Obstetrics and Gynecology, vol. 140, no. 8, pp. 874-879, 1981.

[21] J. H. Levin, F. Z. Stanczyk, and R. A. Lobo, "Estradiol stimulates the secretion of prostacyclin and thromboxane from endometrial stromal cells in culture," Fertility and Sterility, vol. 58, no. 3, pp. 530-536, 1992.

[22] B. Jana, A. Kozłowska, A. Andronowska, and M. JedlińskaKrakowska, "The effect of tumor necrosis factor- $\alpha$ (TNF- $\alpha$ ), interleukin (IL)- $1 \beta$ and IL-6 on chorioamnion secretion of prostaglandins $(\mathrm{PG}) \mathrm{F}_{2 \alpha}$ and $\mathrm{E}_{2}$ in pigs," Reproductive Biology, vol. 8, no. 1, pp. 57-68, 2008.

[23] S.-H. Cho and E.-W. Hwang, "Acupuncture for primary dysmenorrhoea: a systematic review," BJOG, vol. 117, no. 5, pp. 509$521,2010$. 


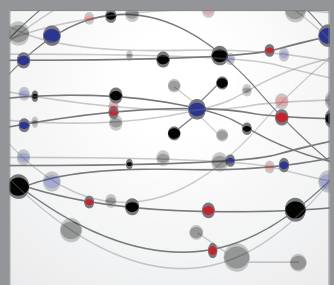

The Scientific World Journal
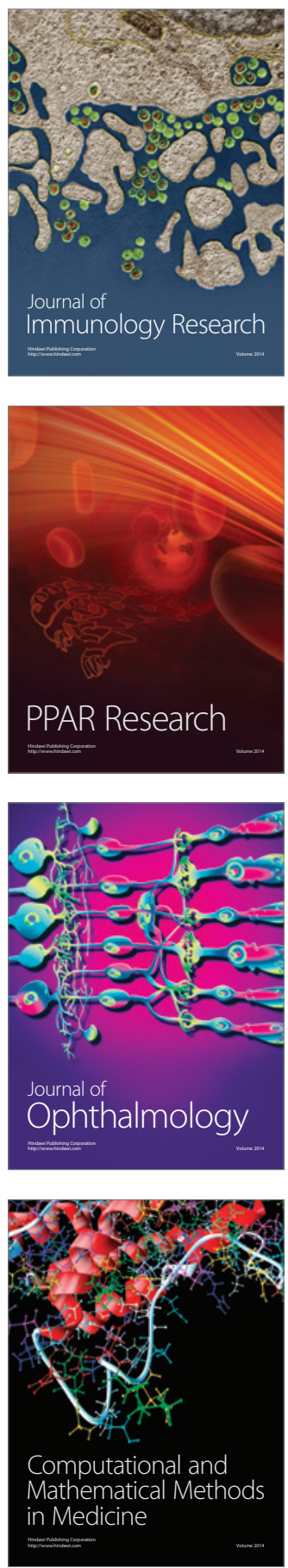

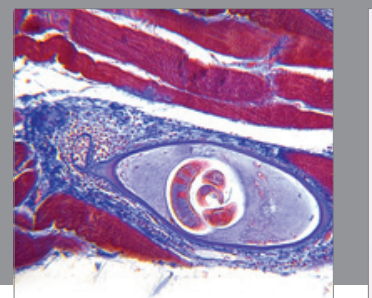

Gastroenterology

Research and Practice
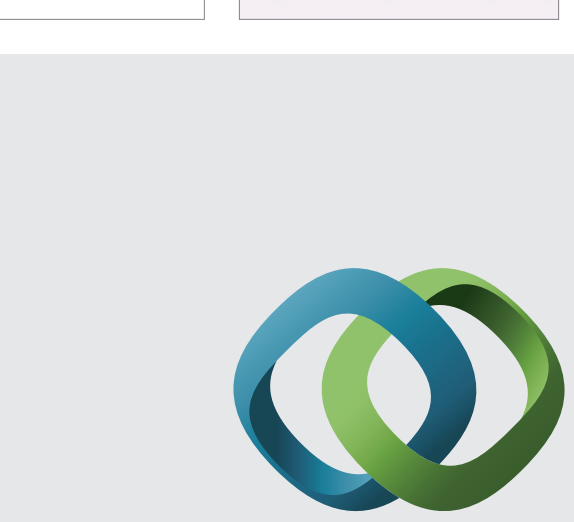

\section{Hindawi}

Submit your manuscripts at

http://www.hindawi.com
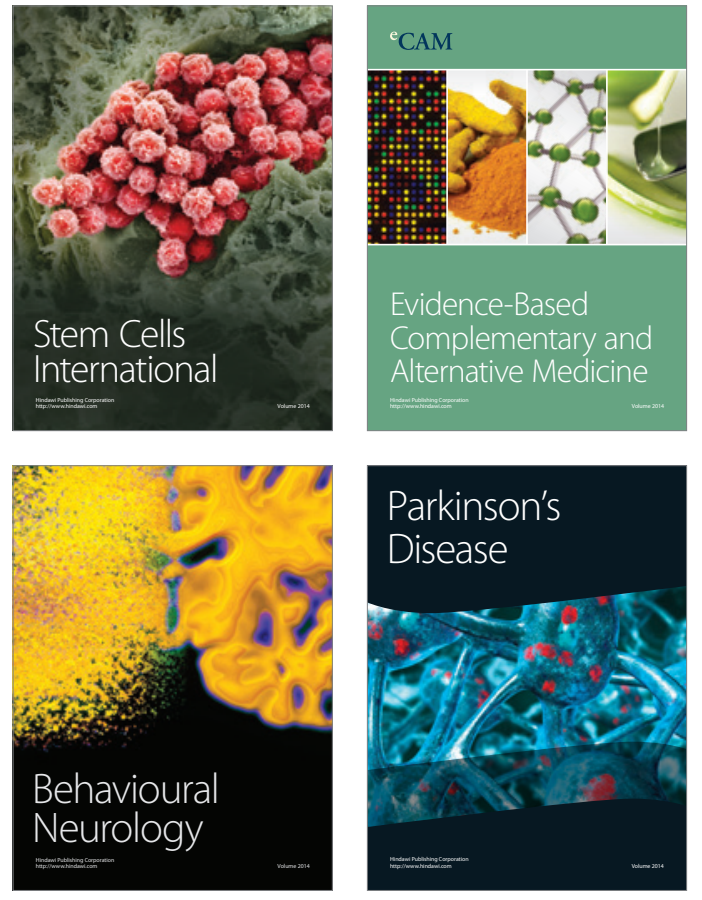
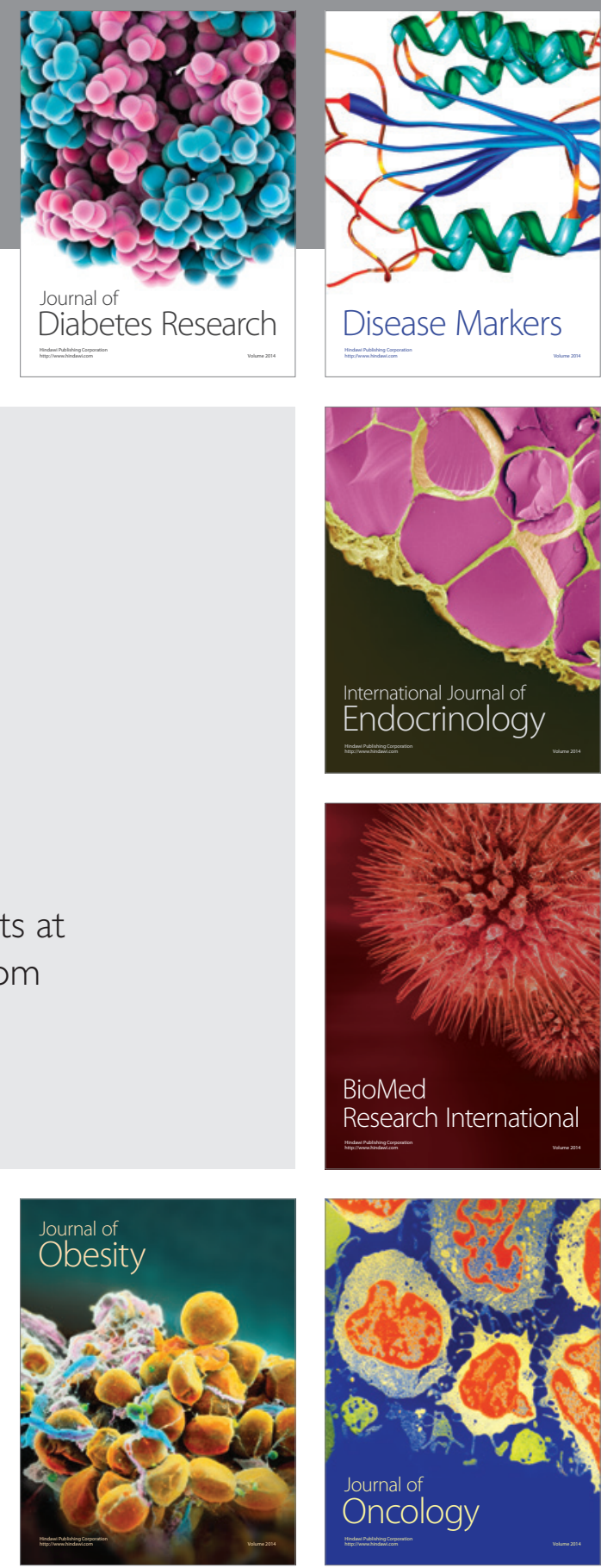

Disease Markers
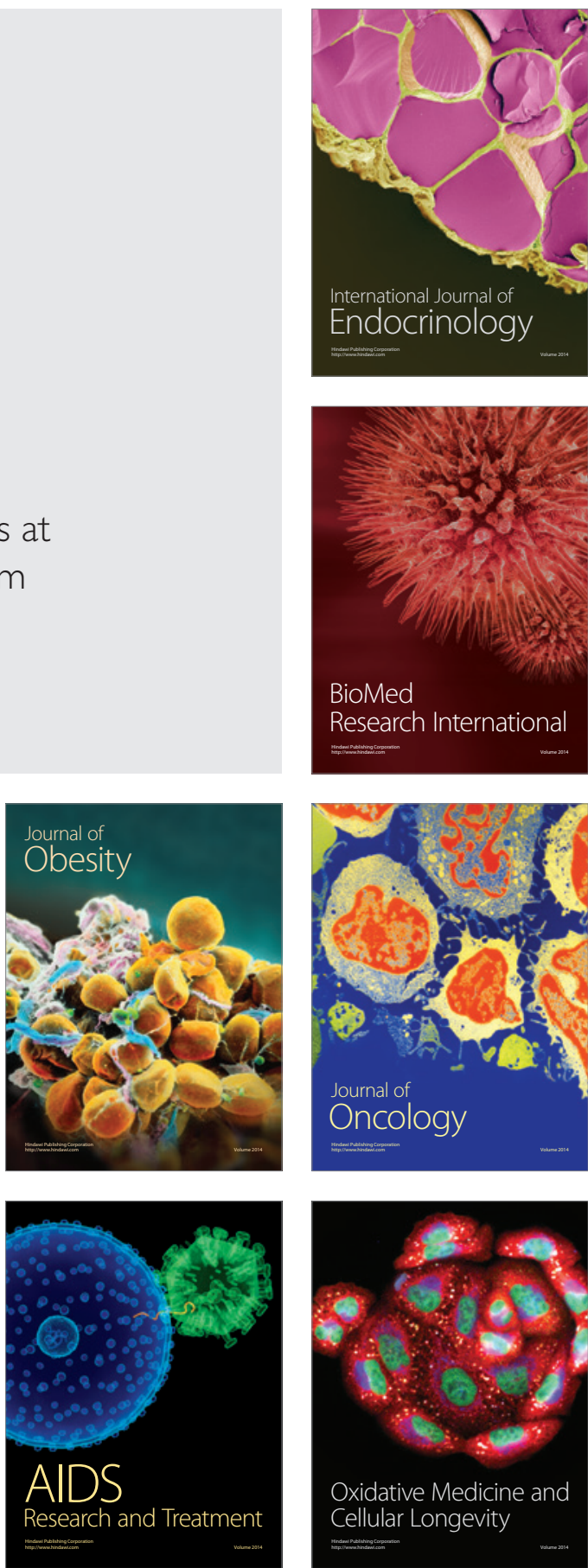